\title{
ON UNIFORMLY BOUNDED SEQUENCES IN ORLICZ SPACES
}

\author{
ERJK J. BALDER
}

A useful result for uniformly bounded sequences of functions in Orlicz spaces is generalised by means of a recent extension of Komlos' theorem. The same generalisation can also be proven differently, by means of Young measures.

\section{A QUESTION}

Let $\left(f_{k}\right)$ be a given sequence of $E$-valued functions in some Orlicz space of functions $((E,\|\cdot\|)$ a Banach space). The following question is of some interest, for instance in connection with certain variational problems on such Orlicz spaces:

QUESTION 1.1. Under which conditions do there correspond to $\left(f_{k}\right)$ a function $f_{*}$ in the Orlicz space and a sequence $\left(g_{n}\right)$ of strongly measurable functions $g_{n}: \Omega \rightarrow E$ such that a.e. in $\Omega$

$$
\begin{gathered}
g_{n}(\omega) \text { is a convex combination of }\left\{f_{k}(\omega): k \geqslant n\right\}, n \in \mathrm{N} \\
\left\|g_{n}(\omega)-f_{*}(\omega)\right\| \rightarrow 0 ?
\end{gathered}
$$

An answer to this question was given by Giner in his thesis. Namely, by [12, Proposition 3.2.2] it is enough for $\left(f_{k}\right)$ to be uniformly bounded as a subset of an Orlicz space whose norming function has certain growth properties. In Giner's framework, which is based on certain decomposition results for the dual of the Orlicz space, it is essential that $E$ is a reflexive Banach space. A special version of Giner's result, also proven by decomposition results, can be found in Levin [14, Corollory 6 of Theorem 7.1] (with the $L^{1}$-space $\mathcal{L}_{E}^{1}$ of $E$-valued integrable functions as the Orlicz space). A slightly different version of these results was recently given by Castaing [9, Theorem 3.4], who proved it by using the so-called biting lemma $[10$, Lemma $C]$ (see also $[8,6,7]$ As one prominent application, we mention the proof of an influential existence result of Levin $[13$, Theorem 1] (see also $[15,14]$ ); interestingly, a direct proof of this existence result by means of the biting lemma is also possible, as was observed by Balder [6].

Received 4th August 1989.

V.F. Gaposhkin is the earliest reference known to me; I am indebted to Frank H. Page for calling it to my attention.

Copyright Clearance Centre, Inc. Serial-fee code: 0004-9729/90 \$A2.00+0.00. 
It is an immediate observation that if $E$ is superreflexive and the Orlicz space is Levin's $\mathcal{L}_{E}^{1}$, then Garling's [11] abstract "strong" infinite-dimensional extension of Komlós' theorem forms a direct answer to the question above. Moreover, this provides much more detailed information than the answers given by Levin and Giner; for finitedimensional $E$ this was already observed by Levin $[14$, p.283] (see also a related remark in $[13$, p.1385] in connection with Levin's existence result).

The point to be made in this note is that even if $E$ is not superreflexive, Balder's recent "weak" infinite-dimensional generalisation of Komlós' theorem [4, 5] still provides an immediate (and superior) answer to Question 1.1. This answer is presented by Theorem 3.1 below.

As an interesting side-remark, we shall observe that Theorem 3.1 can also be proven by means of Young measures [2]. This is a rather unrelated approach; yet the conditions which it needs are precisely the same as those used for the proof by way of Komlós' theorem.

\section{A Result of Komlós TyPE}

We shall formulate a corollary of $[5$, Theorem 2.1], which deals with the following specialisation of the general framework given there. Let $(\Omega, \mathcal{F}, \mu)$ be an arbitrary measure space. Let $(E,\|\cdot\|)$ be a Banach space; this space is supposed to have been equipped with a fixed topology $\tau$, which is not stronger than the norm topology and not weaker than the weak topology $\sigma\left(E, E^{\prime}\right)$. An inf-compact convex integrand on $\Omega \times E$ is defined to be a function $H: \Omega \times E \rightarrow(-\infty,+\infty]$ such that for every $\omega \in \Omega$ the function $H(\omega, \cdot)$ is inf-compact (with respect to the topology $\tau$ ) and convex on $E$. The following result slightly generalises the weak infinite-dimensional extension of Komlós' theorem, introduced in [4, Theorem $\mathrm{A}]$, [5, Theorem 3.2].

THEOREM 2.1. Suppose that $\left(f_{k}\right)$ is a sequence of strongly measurable functions $f_{k}: \Omega \rightarrow E$, for which there exists an inf-compact convex integrand $H: \Omega \times E \rightarrow$ $(-\infty,+\infty]$ such that

$$
\sup _{k \in N} \int_{\Omega}^{*} H\left(\omega, f_{k}(\omega)\right) \mu(d \omega)<+\infty
$$

(here $\int_{\Omega}^{*}$ denotes outer integration over $\Omega$ ) and for certain $\alpha>0$ and $\phi \in \mathcal{L}_{\mathbf{R}}^{1}$

$$
H(\omega, x) \geqslant \alpha\|x\|+\phi(\omega) .
$$

Then there exist a subsequence $\left(f_{m}\right)$ of $\left(f_{k}\right)$ and a strongly measurable function $f_{*}$ : $\Omega \rightarrow E$ such that

$$
\int_{n}^{*} H\left(\omega, f_{*}(\omega)\right) \mu(d \omega)<+\infty
$$


and for every subsequence $\left(f_{m_{i}}\right)$ of $\left(f_{m}\right)$

$$
\frac{1}{n} \sum_{i=1}^{n} f_{m_{i}}(\omega) \stackrel{\tau}{\rightarrow} f_{*}(\omega) \text { as } n \rightarrow \infty \text { a.e. in } \Omega \text {. }
$$

Proof: The proof is entirely similar to the proof of [5, Theorem 3.2], which is entirely based on applying Theorem. 2.1 of [5]. For some details we refer to that proof. The strongly measurable functions $\left(f_{k}\right)$ are norm-separably-valued on $\Omega$, except perhaps for an exceptional set $N$ of measure zero. Define $D:=$ cl co $\cup_{k} f_{k}(\Omega \backslash N)$; then by [5, Lemma 3.1] $(D,\|\cdot\|)$ is a Polish space, so $(D, \tau)$ must be a Suslin space. This takes care of condition (2.2) of [5, Theorem 2.1]. Now $D$ is weakly closed (by the Hahn-Banach theorem), hence also $\tau$-closed. Consequently, instead of the integrand $h$ figuring in the proof of [5, Theorem 3.2], we may now use $h(\omega, x):=H(\omega, x)$ if $x \in D$, and $h(\omega, x):=+\infty$ else, and conclude that $h(\omega, \cdot)$ is sequentially inf-compact for $\tau$, since compact subsets of a Suslin space are metrisable. By force of (2.1), this takes care of condition (2.1) of [5, Theorem 2.1]. Finally, the remaining condition $(B)$ of the latter theorem holds by (2.2). So all conditions of [5, Theorem 2.1] hold when we make the same substitutions as in the proof of [5, Theorem 3.2]. The rest of the proof is exactly as given there.

REMARK 2.2. If instead of requiring $H(\omega, \cdot)$ to be inf-compact, we had required $H(\omega, \cdot)$ to be sequentially inf-compact, the proof by means of $[5$, Theorem 2.1$]$ could have been even more direct.

REMARK 2.3. If instead of requiring $H(\omega, \cdot)$ to be inf-compact, we allow $H(\omega, \cdot)$ to be locally inf-compact (that is, only the intersections of the level sets of $H(\omega, \cdot)$ with closed balls have to be compact), Theorem 2.1 remains trivially valid, in view of (2.2).

REMARK 2.4. Garling [5, Theorem 6] has shown that, if $E$ is superreflexive and if $H(\omega, x):=\|x\|$, the arrow $\stackrel{\tau}{\rightarrow}$ in (2.4) can be replaced by convergence in norm on $\mathrm{E}$ (even though $B(\omega, \cdot):=\|\cdot\|$ is not inf-compact for the norm-topology - unless $\mathrm{E}$ is finite-dimensional, of course).

\section{A General Answer}

As in Section 2, let $E$ be a Banach space, equipped with a topology $\tau$ which is not stronger than the norm topology and not weaker than the weak topology on $E$. Further, in this section $(\Omega, \mathcal{F}, \mu)$ will be a $\sigma$-finite measure space. Let $H: \Omega \times E \rightarrow(-\infty,+\infty]$ be an inf-compact convex integrand. By $\mathcal{L}_{E}^{H}$ we shall denote the set of all strongly measurable functions $f: \Omega \rightarrow E$ such that

$$
\int_{\Omega}^{*} H(\omega, f(\omega)) \mu(d \omega)<+\infty
$$


(In the case of conventional Orlicz spaces, further conditions are imposed on $H$, which allow a seminorm to be associated with the above expression. We shall briefly expound on this below.)

The following result presents a rather general answer to Question 1.1 .

THEOREM 3.1. Suppose that the sequence $\left(f_{k}\right)$ in $\mathcal{L}_{E}^{H}$ satisfies

$$
\sup _{k \in N} \int_{\Omega}^{*} H\left(\omega, f_{k}(\omega)\right) \mu(d \omega)<+\infty
$$

and suppose that for certain measurable functions $a: \Omega \rightarrow(0,+\infty), b: \Omega \rightarrow R$

$$
H(\omega, x) \geqslant a(\omega)\|x\|+b(\omega) .
$$

Then there exist a function $f_{*} \in \mathcal{L}_{E}^{H}$ and a sequence $\left(g_{n}\right)$ of strongly measurable functions such that a.e. in $\Omega$

$$
\begin{gathered}
g_{n}(\omega) \in \operatorname{co}\left\{f_{k}(\omega): k \geqslant n\right\}, \\
\left\|g_{n}(\omega)-f_{*}(\omega)\right\| \rightarrow 0 .
\end{gathered}
$$

Proof: Suppose first that $H$ satifies condition (2.2) of Theorem 2.1. We can then apply Theorem 2.1. This gives us already the desired function $f_{*}$, and from the corresponding subsequence $\left(f_{m}\right)$ we can construct the desired $\left(g_{n}\right)$ in an elementary way. For (2.4) is quickly seen to imply that for arbitrary, fixed $p \in N$

$$
f_{*}(\omega) \in \tau \text {-cl co }\left\{f_{m}(\omega): m \geqslant p\right\} \text { a.e. in } \Omega .
$$

Hence, by the position of the topology $\tau$, the Hahn-Banach theorem implies that for almost every $\omega \in \Omega$

$$
f_{*}(\omega) \in \text { norm-cl co }\left\{f_{m}(\omega): m \geqslant p\right\}=\text { norm-cl corat }\left\{f_{m}(\omega): m \geqslant p\right\} .
$$

Here corat stands for the convex hull over the rationals. Now the set of all rational convex combinations of the functions $f_{m}, m \geqslant p$, is countable; hence we can express it as $\left\{e_{j}: j \in N\right\}$, say. So by (3.6) we have for almost every $\omega \in \Omega$

$$
\left\|f_{*}(\omega)-e_{j}(\omega)\right\|<\frac{1}{p} \text { for some } j \in \mathrm{N} .
$$

By forming a suitable countable partitioning of $\Omega$ it is thus evident that there exists a function $g_{p}$, which coincides with an element from $\left\{e_{j}\right\}$ on each set of the partitioning, for which

$$
\left\|f_{*}(\omega)-g_{p}(\omega)\right\|<\frac{1}{p} \text { a.e. in } \Omega .
$$


This concludes the construction of the sequence $\left(g_{n}\right)$, which evidently satisfies (3.4)-(3.5). It remains to show how condition (2.2), used so far, can be replaced by (3.3). This is also simple, for by $\sigma$-finiteness of $(\Omega, \mathcal{F}, \mu)$, there exists a strictly positive function $\hat{\phi} \in \mathcal{L}_{\mathbf{R}}^{1}$. Define now for $n \in N$

$$
\Omega_{n}:=\left\{\omega \in \Omega: a(\omega) \geqslant \frac{1}{n}, b(\omega) \geqslant-n \hat{\phi}(\omega)\right\}, n \in N .
$$

Then $\left(\Omega_{n}\right)$ increases to $\Omega$, and, with respect to each $\Omega_{n}$, condition (3.3) turns into (2.2). By a rather obvious diagonal sequence argument, which uses (2.4) on the successive parts $\Omega_{n}, n \in N$, we arrive at the desired functions for which (3.4)-(3.5) then hold. (Note that by the monotone convergence theorem, applied in connection with the successive statements (2.3), $f_{*}$ is seen to belong to the Orlicz space).

Let us briefly indicate the connection with the earlier versions of Theorem 3.1, referenced in section 1. First, in the conventional framework of Orlicz spaces, which figures in Giner's work [12], $B$ is a so-called Young function, that is, an $\mathcal{F} \times \mathcal{B}(E)$ measurable function $H: \Omega \times E \rightarrow[0,+\infty]$ such that for (almost) every $\omega \in \Omega$ the function $H(\omega, \cdot)$ is lower semicontinuous, convex, coercive, even and equal to zero in 0 . In this way there corresponds a natural seminorm to (3.1), which is defined on the vector space generated by $\mathcal{L}_{E}^{H}$ (see [12] for details). What is relevant for the present note is that the above coercivity property implies that Giner's $B(\omega, \cdot)$ is inf-compact on $E$ for $\tau:=\sigma\left(E, E^{\prime}\right)$ (recall that $E$ is reflexive in Giner's work). Moreover, by [12, Theorem 1.1.10(iii)] any Young function $B$ automatically satisfies an inequality which is stronger than our condition (3.3). Of course, the above remarks apply a fortiori to the less general framework employed by Levin in [14]. Finally, Castaing's result in [9, Theorem 3.4] is also clearly contained in our Theorem 3.1. For in his main condition the values $\left(f_{k}(\omega)\right)$ are pointwise contained in some closed convex set $L(\omega)$, whose intersection with every closed ball around 0 is $\sigma\left(E, E^{\prime}\right)$-compact. Thus, if we set $H(\omega, x):=\|x\|$ if $x \in L(\omega)$ and $H(\omega, x):=+\infty$ otherwise, then for the integrand $H$ all conditions of Theorem 3.1 turn out to hold (see also a similar point made in Remark 2.2).

\section{Connections with Young Measures}

It is perhaps of some interest to point out that Theorem 3.1 can also be proven by a very basic argument involving Young measures. Since this observation serves only as a side-remark, we shall not recapitulate here the essentials of Young measure theory. Instead, we refer the reader to $[1,2,3,16]$.

The sketch of the proof is as follows. First, note that we may suppose $\mathrm{E}$ to be normseparable with no loss of generality (see the proof of Theorem 2.1 with regard to the 
almost separably-valuedness of $\left(f_{k}\right)$ ). So now $E$ is a completely regular Suslin space, and fits into the framework for Young measure theory. Condition (3.1) constitutes for $\left(f_{k}\right)$ a tightness condition in the sense of $[1,2,3]$ (also called B-tightness in [16]). Hence, by the extension of Prohorov's theorem for Young measures (which itself can be proven, among other ways, by the weak extension of Komlós' theorem [5, Theorem 5.1]) there exists a subsequence $\left(f_{m}\right)$ which converges narrowly (in the sense of Young measures) to some Young measure $\delta_{*}$, say. It is well-known (see the above references) that then

$$
\delta_{*}(\omega)\left(\operatorname{Ls}\left(f_{m}(\omega)\right)\right)=1 \text { a.e. in } \Omega,
$$

where $\operatorname{Ls}\left(f_{m}(\omega)\right)$ denotes the set of all limit points of the sequence $\left(f_{m}(\omega)\right)$. Further, one then has

$$
\int_{\Omega}^{*}\left[\int_{E} H(\omega, x) \delta_{*}(\omega)(d x)\right] \mu(d \omega)<+\infty
$$

so by inequality (3.2) the barycenter $f_{*}(\omega)$ of the probability measure $\delta_{*}(\omega)$, defined as

$$
f_{*}(\omega):=\int_{E} x \delta_{*}(\omega)(d x)
$$

exists a.e. in $\Omega$. By (4.1), then for every $p \in N$

$$
f_{*}(\omega) \in \operatorname{cl} \text { co }\left\{f_{m}(\omega): m \geqslant p\right\} \text { a.e. in } \Omega,
$$

so we can continue from here onwards to (3.3) by following the argument given in the proof of Theorem 3.1. Note that until now all steps in this section have been made without requiring the convexity of the functions $H(\omega, \cdot), \omega \in \Omega$. At this juncture, however, we invoke such convexity to deduce (3.1) from (4.2) by Jensen's inequality. This finishes our sketch of the proof. Its potential to deal with nonconvexities should make this approach by Young measures an appealing alternative, for it is well-known that to take barycenters and convex hulls is not the only way to associate meaningful functions with $\left(f_{k}\right)$ (see [2] for instances of this). On the other hand, as Balder has shown in [5], much of the subject of Young measures (including all steps taken in this section) can also be approached by the weak extension of Komlós' theorem.

\section{EPILOGUE}

In this note we have shown how Theorem 3.1 generalises the answers to Question 1.1 as given by Giner [12, Proposition 3.2.2], Levin [14, Corollory 6 of Theorem 7.1] and Castaing [9, Theorem 3.4]. Since Theorem 3.1 was shown to follow elementarily 
from the weak extension of Komlós' theorem, achieved in $[4,5]$, the real answer to the question is already contained in Komlós' theorem. Obviously, this holds all the more forcefully if $\mathrm{E}$ is a superreflexive Banach space, in view of Garling's strong infinitedimensional extension of Komlós' theorem, which we discussed in Remark 2.3. See also our comments in Section 1. As a side-remark, we have also pointed out how Theorem 3.1 ties in with our earlier results in Young measure theory.

\section{REFERENCES}

[1] E.J. Balder, 'A general approach to lower semicontinuity and lower closure in optimal control theory', SIAM J. Control Optim. 22 (1984), 570-598.

[2] E.J. Balder, 'Fatou's lemma in infinite dimensions', J. Math. Anal. Appl. 116 (1988), 450-465.

[3] E.J. Balder, 'On Prohorov's theorem for transition probabilities', Sém. Anal. Convexe 19 (1989), 9.1-9.11.

[4] E.J. Balder, 'Infinite-dimensional extension of a theorem of Komlos', Probab. Theory Related Fields 81 (1989), 185-188.

[5] E.J. Balder, 'New sequential compactness results for spaces of scalarly integrable functions', J. Math. Anal. Appl. (1990) (to appear).

[6] E.J. Balder, 'Short proof of an existence result of V.L. Levin', Bull. Polish Acad. Sci. Math. (to appear).

[7] J.M. Ball and F. Murat, 'Remarks on Chacon's biting lemma', preprint Analyse Numérique, Université Pierre et Marie Curie, Paris, 1988.

[8] J.K. Brooks and R.V. Chacon, 'Continuity and compactness of measures', Adv. in Math. 37 (1980), 16-26.

[9] C. Castaing, 'Sur la décomposition de Slaby. Applications aux problèmes de convergence en probabilités, économie mathématique, théorie du contrôle, minimisation', Sém. Anal. Convexe 19 (1989) (to appear).

[10] V.F. Gaposhkin, 'Convergence and limit theorems for sequences of random variables', Theory Prob. and Appl. 17 (1972), 379-400.

[11] D.J.H. Garling, 'Subsequence principles for vector-valued random variables', Math. Proc. Cambridge Philos Soc. 86 (1979), 301-311.

[12] E. Giner, Espaces Integraux de Type Orlicz'(Thèse, Université des Sciences et Techniques du Languedoc, Perpignan, 1977).

[13] V.L. Levin, 'Extremal problems with convex functionals that are lower semicontinuous with respect to convergence in measure Dokl. Akad. Nauk SSSR' 224, pp. 1256-1259. Sov. Math. Dokl. 16 (1976), 1384-1388).

[14] V.L. Levin, Convex Analysis, (in Russian), Nauka, Moscow, (1985).

[15] M. Valadier, 'Convergence en mesure et optimisation (d'après Levin)', Sém. Anal. Conveze 6 (1976), 14.1-14.9.

[16] M. Valadier, Young Measures (CDME Lecture Notes, 1989). (to appear). 
Mathematical Institute University of Utrecht

Utrecht

Netherlands 